\title{
Equilibrium States for Classical Systems
}

\author{
C. Gruber \\ Laboratoire de Physique Théorique, Ecole Polytechnique Fédérale, Lausanne, Switzerland
}

\author{
J. L. Lebowitz` \\ Belfer Graduate School of Science, Yeshiva University, New York, N.Y., USA
}

Received July 18, 1974

\begin{abstract}
It is shown that the Dobrushin-Lanford-Ruelle equations for the probability measure $\mu$, and the Kirkwood-Salsburg type equations for the lattice or continuum correlation functions $\varrho$, and for the spin correlation functions $\sigma$, are essentially equivalent for all $\varrho, \sigma$, and $\mu$ satisfying certain boundedness conditions. It is also noted that the lattice equations are identical to the equations for the stationary states of a certain Markoff process. This extends previous results of Ruelle, Brascamp and Holley who proved some of these equivalences for states.
\end{abstract}

\section{Introduction}

The equilibrium states of an infinite classical lattice system can be specified by various means $[1-5]$, e.g.:

1. States defined by a probability measure $\mu$ which is a solution of a linear equation called the "Equilibrium Equation". These states are called "Equilibrium States".

2. States defined by the solutions of a set of equations for the correlation function $\varrho(X)$ (lattice gas language).

3. States defined by the solutions of a set of equations for the expectation values $\sigma(X)$ (spin language).

It is known that 1 . implies 2 . $[2,3,4]$ and conversely any state whose correlations satisfy 2. is an Equilibrium State. In this note we point out that the Equilibrium Equations and the equations for $\varrho(X)$ or $\sigma(X)$ are equivalent in an even stronger sense, i.e. their solutions, with suitable boundedness properties, even if they do not define states are also equivalent. Indeed the different equations are expressions of the same relation using different basis for the observables.

\section{Lattice Systems}

We consider a spin $\frac{1}{2}$ classical lattice system defined on a lattice $\mathscr{L}$; the algebra $\mathfrak{U}$ of observables is the algebra of continuous functions on the compact set [5]

$$
\Gamma=\prod_{x \in \mathscr{L}}\{0,1\}_{x}=\mathscr{P}(\mathscr{L}) .
$$

* Research supported in part by AFOSR Grant No. 73-2430A. 
A state $\omega$ is defined by a probability measure on the Borel sets of $\Gamma$; it specifies for any finite subset $\Lambda \subset \mathscr{L}$ and any $X \subset \Lambda$, a measure $\mu_{\Lambda}(X ; d Y)$ on the compact set $\mathscr{P}(\mathscr{L} / \Lambda)$ representing the probability that a set $Z \subset \mathscr{L}$ is of the form $Z=X \cup Y$ with $Y \subset \mathscr{L} / \Lambda$ and $Y \in d Y$. For any observable $A \in \mathfrak{A}$ we have

$$
\omega(A)=\lim _{\boldsymbol{\Lambda} \rightarrow \mathscr{L}} \sum_{X \subset \boldsymbol{\Lambda}} \mu_{\Lambda}(X) A(X)
$$

where $A(X)$ means, now, the value of $A$ on the element of $\mathscr{P}(\mathscr{L})$ which is empty outside $X$ and

$$
\mu_{\Lambda}(X)=\int_{\mathscr{P}(\mathscr{L} / \Lambda)} \mu_{\Lambda}(X ; d Y) .
$$

The energy of the configuration $X$ is given by:

$$
H(X)=\sum_{T \subset X} \Phi(T)
$$

where

$$
\sup _{x \in \mathscr{L}}\left\{\sum_{X \ni x}|\Phi(X)|\right\}<\infty
$$

The interaction is said to have finite range if for any $x \in \mathscr{L}$, there exists a finite set $\Delta_{x}$ such that $\Phi(x \cup X)=0$ if $X \nsubseteq \Delta_{x}$.

The state $\omega$ is said to be an "Equilibrium State" with respect to the interaction $\Phi$ if it is a solution of the equilibrium equation [2]:

$$
\mu_{\Lambda}(x \cup X ; d Y)=e^{-\beta[H(x \cup X \cup Y)-H(X \cup Y)]} \mu_{\Lambda}(X ; d Y)
$$

for all finite $\Lambda, x \in \Lambda, X \subset \Lambda / x$.

Introducing the function $E_{x}$ by:

$$
2 E_{x}(R)=H(x \cup R)-H(R)
$$

(2.3) can also be written $[2,4]$ in the form

$$
\mu_{\Lambda}(x \cup X)=\int_{I_{\Lambda}(X)} \mu_{\Lambda}(X ; d Y) e^{-2 \beta E_{x}(X \cup Y)}
$$

and for finite range potential,

$$
\mu_{\Lambda}(x \cup X)=e^{-2 \beta E_{x}(X)} \mu_{\Lambda}(X)
$$

for all finite $\Lambda, x \cup X \subset \Lambda, \Delta_{x} \subset \Lambda$.

It is customary to introduce the following family of observables whose linear span are dense in $\mathfrak{U}$ :

$$
\begin{array}{ll}
\varrho_{R}=\prod_{r \in R} \varrho_{R}, \quad \varrho_{r}(X)=\left\{\begin{array}{lll}
1 & \text { if } & r \in X \\
0 & \text { if } & r \notin X
\end{array}\right. \\
\sigma_{R}=\prod_{r \in R} \sigma_{r}, \quad \sigma_{r}=1-2 \varrho_{r}, \quad R \in \mathscr{P}_{f}(\mathscr{L})
\end{array}
$$

The correlation functions $\varrho$ and $\sigma$ are defined by:

Moreover

$$
\varrho(X)=\omega\left(\varrho_{X}\right), \quad \sigma(X)=\omega\left(\sigma_{X}\right)
$$

$$
\mu_{\Lambda}(X)=\omega\left(\varrho_{X} \prod_{r \in \Lambda / X}\left(1-\varrho_{r}\right)\right)
$$


The connection between the different descriptions is obtained by means of the operators $\mathscr{A}_{A}, \mathscr{F}_{A}$ together with their "inverse" $\mathscr{A}_{A}^{-1}, \mathscr{F}_{A}^{-1}$, operators defined on the space of bounded functions $\varphi$ on $\mathscr{P}_{f}(\mathscr{L})$ by:

$$
\begin{array}{ll}
\left(\mathscr{A}_{\Lambda} \varphi\right)(X)=\chi_{\Lambda}(X) \sum_{Y \subset \Lambda / X} \varphi(X \cup Y) & \left(\mathscr{A}_{\Lambda}^{-1} \varphi\right)(X)=\chi_{\Lambda}(X) \sum_{Y \subset \Lambda / X}(-1)^{|Y|} \varphi(X \cup Y) \\
\left(\mathscr{F}_{\Lambda} \varphi\right)(X)=\chi_{\Lambda}(X) \sum_{Y \subset A} \sigma_{Y}(X) \varphi(Y) \quad\left(\mathscr{F}_{\Lambda}^{-1} \varphi\right)(X)=\chi_{\Lambda}(X) 2^{-|\Lambda|} \sum_{Y \subset A} \sigma_{Y}(X) \varphi(Y)
\end{array}
$$

where $\sigma_{Y}(X)=(-1)^{|X \cap Y|}$ and $|S|=$ number of sites in $S$, and which satisfy:

$$
\mathscr{A}_{\Lambda} \mathscr{A}_{A}^{-1}=\mathscr{A}_{A}^{-1} \mathscr{A}_{A}=\chi_{A} \quad \mathscr{F}_{\Lambda} \mathscr{F}_{A}^{-1}=\mathscr{F}_{A}^{-1} \mathscr{F}_{A}=\chi_{A} .
$$

The connection between the different descriptions is then given by:

$$
\mu_{A}=\mathscr{A}_{\Lambda}^{-1} \varrho=\mathscr{F}_{\Lambda}^{-1} \sigma
$$

From the above definitions it follows at once that the correlation functions $\varrho$, and $\sigma$ define a state if and only if:

$$
\varrho(\emptyset)=1 \text { and } \mathscr{A}_{\Lambda}^{-1} \varrho \geqq 0 \text { for all finite } \Lambda \subset \mathscr{L}
$$

or

$$
\sigma(\emptyset)=1 \text { and } \mathscr{F}_{A}^{-1} \sigma \geqq 0 \text { for all finite } \Lambda \subset \mathscr{L}
$$

\section{Correlation Equations and Equivalence}

It has been shown $[2,4,7]$ that for any equilibrium state $\omega$ the functions $\varrho$ is a solution of the following "Gallavotti-Miracle" (GM) Equation

$$
\varrho(\emptyset)=1, \varrho(X)=\sum_{T \subset \mathscr{L} / x} K(x ; X / x ; T)\{\varrho(T \cup X / x)-\varrho(T \cup X)\}=\left(\mathscr{K}_{x} \varrho\right)(X)
$$

where $K(x ; X ; T)=\Sigma_{Z \subset T}(-1)^{|T|-|Z|} e^{-2 \beta E_{x}(X \cup Z)}$. The $\mathrm{GM}$ equation can be written in an obvious way as a linear equation on the space $\mathscr{D}$ of bounded functions $\psi$ on $\mathscr{P}_{f}(\mathscr{L})$

$$
\varrho=1+\mathscr{K} \varrho, \quad \text { with } \quad 1(X)=\delta_{X, \emptyset}
$$

We shall also define $\mathscr{D}_{s} \subset \mathscr{D}$ as the space of "symmetric functions" $\psi$ s.t.

$$
\left(\mathscr{K}_{x_{i}} \psi\right)(X)=\left(\mathscr{K}_{x_{j}} \psi\right)(X) \text { for all } x_{i}, x_{j} \in X .
$$

We shall show below that for any equilibrium state $\omega$, the function $\sigma$ is a solution of the equation:

$$
\begin{aligned}
\omega\left(\sigma_{x} \sigma_{X}\right) & =\omega\left(\sigma_{X} \tanh \beta E_{x}\right) \\
\omega\left(\sigma_{\natural}\right) & =1
\end{aligned}
$$

for any $x \notin X$. This equation is valid for any interaction such that $\tanh \beta E_{x}$ is a continuous function on $\Gamma$. 
This equation was studied in [7] and for finite range interactions becomes:

$$
\left\{\begin{array}{l}
\sigma(\emptyset)=1 \\
\sigma(x \cup X)=\sum_{R \neq x} a(x ; R) \sigma(X R) ; \quad X R=X \cup R-X \cap R
\end{array}\right.
$$

where

$$
a(x ; R)=2^{-\left|\Delta_{x}\right|} \sum_{S \ngtr x} \sigma_{s}(R) \tanh \left[\beta E_{x}(R)\right]
$$

Lemma 1. For finite range interaction and any $\psi \in \mathscr{D}_{\mathrm{s}}$

$$
\left(\mathscr{A}_{\Lambda}^{-1} \mathscr{K} \psi\right)(x \cup X)=e^{-2 \beta E_{x}(X)}\left(\mathscr{A}_{\Lambda}^{-1} \psi\right)(X),
$$

for $X \subset \Lambda / x$, and $\Delta_{x} \subset \Lambda$.

Lemma 2. For interactions satisfying the condition (2.2) any bounded solution $\varrho$ of GM Equation yields a solution $\mu_{\Lambda}=\mathscr{K}_{\Lambda}^{-1} \varrho$ of the Equilibrium Eq. (2.4) or (2.5) satisfying the normalization and compatibility conditions [2] and conversely.

Lemma 3. i) For complex valued interactions such that $\tanh \beta E_{x}$ is a continuous function on $\Gamma$ for all $x$ in $\mathscr{L}$, any bounded solution $\sigma$ of Eq. (3.2) or (3.3) defines a solution $\mu_{\Lambda}=\mathscr{F}_{\Lambda}^{-1} \sigma$ of the Equilibrium Eq. (2.4) or (2.5) satisfying the normalization and compatibility conditions and conversely.

ii) Under the same conditions on the interactions, any solution of Eq. (3.2) such that $\mathscr{F}_{\Lambda}^{-1} \sigma \geqq 0$ for all $\Lambda$ defines an Equilibrium State and conversely.

Proof. Lemma 1 follows from the definitions of $\mathscr{A}_{A}$ and $\mathscr{K}$. For finite range potential Lemma 2 is an immediate consequence of Lemma 1, while Lemma 3 follows from the following argument:

For any finite $\Lambda$ such that $X \subset \Lambda / x, \Delta_{x} \subset \Lambda$, Eq. (3.2) together with the identity

$$
\sigma(x \cup X)=\left(\mathscr{F}_{\Lambda} \mathscr{F}_{\Lambda}^{-1} \sigma\right)(x \cup X)=\left(\mathscr{F}_{\Lambda} \mu_{\Lambda}\right)
$$

yields :

$$
\sum_{Y \subset \Lambda} \mu_{\Lambda}(Y) \sigma_{x}(Y) \sigma_{X}(Y)=\sum_{Y \subset \Lambda} \mu_{\Lambda}(Y) \tanh \left[\beta E_{x}(Y)\right] \sigma_{X}(Y)
$$

i.e.

$\sum_{Y \subset \Lambda / x}\left[\mu_{\Lambda}(Y)-\mu_{\Lambda}(x \cup Y)\right] \sigma_{X}(Y)=\sum_{Y \subset \Lambda / x}\left[\mu_{\Lambda}(Y)+\mu_{\Lambda}(x \cup Y)\right] \tanh \left[\beta E_{x}(Y)\right] \sigma_{X}(Y)$

this equality being true for all $X \subset \Lambda / x$, we obtain:

$$
\mu_{\Lambda}(x \cup Y)=\frac{1-\tanh \beta E_{x}(Y)}{1+\tanh \beta E_{x}(Y)} \mu_{\Lambda}(Y)=e^{-2 \beta E_{x}(Y)} \mu_{\Lambda}(Y)
$$

which is the Equilibrium Equation Eq. (2.5). The converse statement is straightforward. The generalization to infinite range interactions is also straightforward. 
We notice at once that for finite lattice $\mathscr{L}$, Lemma 1 yields

$$
\left(\mathscr{A}_{\mathscr{L}}^{-1} \mathscr{K} \psi\right)(x \cup X)=e^{-2 \beta E_{x}(X)}\left(\mathscr{A}_{\mathscr{L}}^{-1} \psi\right)(X) .
$$

But $\psi$ in $\mathscr{P}_{s}$ implies that the L.H.S. of this equation is a symmetric function in the variables $(x \cup X)$, therefore $e^{-2 \beta E_{x}(X)}\left(\mathscr{A}_{\mathscr{\varphi}}^{-1} \psi\right)(X)$ must be symmetric in $(x \cup X)$ for any $\psi$ in $\mathscr{D}_{s}$; this condition is sufficient to obtain explicitly the space $\mathscr{D}_{s}$. This remark is in fact the starting point of Pastur's [6] analysis of the "KirkwoodSalsburg" operator.

To conclude this section we shall consider systems such that:

i) $\mathscr{L}=\mathbb{Z}^{v}$

ii) $\Phi(R)=\Phi(R+a) \quad \forall a \in \mathbb{Z}^{v}$

iii) $\Phi$ has finite range.

For such systems and for translationally invariant states, Lemma 3 appears as a direct consequence of a result obtained by R. Holley [8]. Indeed Holley has studied a Markoff process for such systems which implies that:

$\frac{d}{d t} \mu_{\Lambda}(X ; t)=\sum_{Y \subset M / A} \sum_{x \in A} e^{\beta\left(E_{x} \sigma_{x}\right)(X Y)}\left[\mu_{M}(X Y x)-e^{-2 \beta E_{x}(X Y) \sigma_{x}(X)} \mu_{M}(X Y)\right]$

where

$$
X Y=X \cup Y-X \cap Y
$$

and $\Lambda$ and $M$ are cubes such that $\Delta_{x} \subset M$ for all $x \in \Lambda$. For such a process it follows that the evolution of $\omega_{t}\left(\sigma_{X}\right)$ is given by:

$$
\frac{d}{d t} \omega_{t}\left(\sigma_{X}\right)=-2 \sum_{x \in X} \omega_{t}\left(\sigma_{X} \cosh \beta E_{x}\left[1-\sigma_{x} \tanh \beta E_{x}\right]\right)
$$

We thus see immediately that any state $\omega$ solution of Eq. (3.2) satisfies $\frac{d}{d t} \omega_{t}=0$; using Holley's result we can thus conclude that any translationally invariant solution $\sigma$ of Eq. (3.2) such that $\mathscr{F}_{A}^{-1} \sigma \geqq 0$ defines an equilibrium state.

Moreover it is also a direct consequence of Eq. (2.5) that every equilibrium state is a stationary state for the Markoff process investigated by Holley.

\section{Continuous Systems}

The preceding discussion can be extended to the case of continuous systems, with many-body potentials, i.e. any solution $\varrho$ of Kirkwood-Salsburg equation such that $|\varrho(X)|<\xi^{|X|}$ yields a solution of the Equilibrium Equation satisfying the normalization and compatibility conditions. This is an extension of the results of D. Ruelle [11] who showed, in case of two-body interactions, that if $\omega$ is a state such that the induced measure $\mu$ is tempered, then the correlation function is a solution of Kirkwood-Salsburg equation if and only if $\mu$ is a solution of the equilibrium equation; moreover in this case $|\varrho(X)| \leqq \xi^{|X|}$. We shall limit the discussion to finite range interactions only. 
We consider a classical continuous system of identical particles in $\mathbb{R}^{v}$; the energy of the configuration $\left(x_{1}, \ldots, x_{n}\right)=X$ is

$$
U(X)=\sum_{T \subset X} \Phi(T)
$$

where the interactions $\Phi$ have finite range, $\Phi(x \cup X)=0$ if $\left|x_{i}-x\right|>R$ for some $x_{i} \in X$ and satisfy the condition $2 E_{x}(X)=U(x \cup X)-U(X) \geqq-|X| B$. Let us remark that this last condition is in particular satisfied by ultrastable potential of finite type [9]. The operators $\mathscr{A}_{\Lambda}, \mathscr{A}_{\Lambda}^{-1}$ are defined on the space of symmetric functions by:

$$
\left(\mathscr{A}_{\Lambda} \varphi\right)(X)=\chi_{\Lambda}(X) f_{\Lambda} d Y \varphi(X \cup Y) \quad \text { where } f_{\Lambda} d Y=\sum_{k=0}^{\infty} \frac{1}{k !} \int d y_{1} \ldots d y_{k}
$$

and

$$
\left(\mathscr{A}_{\Lambda}^{-1} \varphi\right)(X)=\chi_{\Lambda}(X) f_{\Lambda} d Y(-1)^{|Y|} \varphi(X \cup Y) .
$$

We have $\mathscr{A}_{A} \mathscr{A}_{A}^{-1}=\mathscr{A}_{A}^{-1} \mathscr{A}_{A}=\chi_{A}$ on the subspace of functions $\varphi$ such that $|\varphi(X)| \leqq \xi^{|X|}$, for some $\xi \geqq 0$. Assuming that the correlation functions $\varrho$ satisfies $|\varrho(X)| \leqq \xi^{|X|}$ the family of distribution functions $\mu_{\Lambda}$ is defined for any bounded $\Lambda \subset \mathbb{R}^{v}$ by:

$$
\mu_{\Lambda}=\mathscr{A}_{\Lambda}^{-1} \varrho \text { and } \chi_{\Lambda} \varrho=\mathscr{A}_{\Lambda} \mu_{\Lambda} \text {. }
$$

We shall say that $\varrho$ is a solution of "Kirkwood-Salsburg Equation" if it satisfies [9]:

$$
\left\{\begin{array}{l}
\varrho(\emptyset)=1 \\
\varrho(x \cup X)=f d Y K(x ; X ; Y) \varrho(X \cup Y)
\end{array}\right.
$$

with $K(x ; X ; Y)=\Sigma_{Z \subset Y}(-1)^{|Y|-|Z|} e^{-2 \beta\left[E_{x}(X \cup Z)\right]}$. Note that $K(x ; X ; Y)=0$ if $|x-y|>R$ for some $y \in Y$.

Theorem 3. i) For interactions satisfying the above conditions, any solution $\varrho$ of Kirkwood-Salsburg equation such that $|\varrho(X)|<\xi^{|X|}$, defines a solution $\left|\mu_{\Lambda}=\mathscr{A}_{\Lambda}^{-1} \varrho\right|$ of the "Equilibrium Equation":

$$
\mu_{\Lambda}(x \cup X)=e^{-\beta[U(x \cup X)-U(X)]} \mu_{\Lambda}(X)
$$

for all bounded $\Lambda \supset x \cup X$ such that $d(x, \partial \Lambda)>R$. Moreover $\mu_{\Lambda}$ satisfies the normalization and compatibility conditions.

ii) Conversely any solution of the Equilibrium Equation Eq. (4.3) which satisfies the normalization and compatibility conditions and such that $\left|\mu_{\Lambda}(X)\right| \leqq_{\Lambda}|X|$, defines a solution of Kirkwood-Salsburg Equation.

Proof. The proof is formally the same as for lattice systems. To conclude this discussion we notice that it follows from Eq. (3.3) that:

$$
\varrho(x \cup Y)=f_{\Lambda} d Z e^{-\beta[U(x \cup Y \cup Z)-U(Y \cup Z)]} \mu_{\Lambda}(Y \cup Z)
$$

for any $\Lambda \subset \mathbb{R}^{v}$ such that $d\left(x_{i}, \partial \Lambda\right)>C_{b}$ from which follows that the induced measure satisfies the Equilibrium Equation introduced by Ruelle in [11]. 


\section{Conclusions}

We have seen that the different types of equations usually considered in equilibrium statistical mechanics yield equivalent definitions of "Equilibrium States". It should be remarked however that these Equilibrium Equations may have solutions which do not define a state $[7,12,13]$. As we have seen, any solution of the equation for $\sigma$ (resp. $\varrho$ ) such that $\mathscr{F}_{\Lambda}^{-1} \sigma \geqq 0$ (resp. $\mathscr{A}_{\Lambda}^{-1} \varrho \geqq 0$ ) defines a state; it would be however of interest to find some more explicit conditions on the space of solutions defining a state. A necessary condition is given for example by $|\sigma(X)| \leqq 1$; it is not known under what conditions such a condition could be sufficient.

A knowledge of some such relatively simple conditions on $\sigma(X)$ might be helpful for answering the question; what are the number of equilibrium states of a spin system for a given value of the thermodynamic parameters $\beta$ and (the magnetic field) $h$ ? Consider for concreteness the case $\mathscr{L}=\mathbb{Z}^{v}$ and let the spin interact with a translation invariant ferromagnetic pair potential only. We know [12] that for $h \neq 0$, all $\beta$, and for $h=0, \beta<\beta_{c}$ there is only one equilibrium state; $\beta_{c}$ is the reciprocal of the temperature where spontaneous magnetization sets in. It is also known that for $h=0$, and $\beta \geqq \beta^{\prime}, \beta^{\prime}$ depending on the interaction but always $\beta^{\prime}>\beta_{c}$ there are at least two extremal equilibrium states. These states correspond to the infinite volume limit of states with + or - boundary conditions. Consider now Eq. (3.2) for this system with $h=0$; it breaks up into two sets of equations, one for the even and one for the odd correlations. For $\beta<\beta_{c}$ there is only obe solution of (2.11) which defines a state while for $\beta>\beta_{c}$ there are many (any combination of the two extremal ones). The question now arises of whether these new states yield new solutions of (3.2) for $\beta<\beta_{c}$ or not. In the first case there is a "bifurcation" of the solutions of (3.3) at $\beta=\beta_{c}$, while in the second case the solutions of (3.3) for $\beta>\beta_{c}$ are a continuation of solutions for $\beta<\beta_{c}$ which however do not define a state for $\beta<\beta_{c}$ but do so for $\beta>\beta_{c}$.

We remark that in the space of bounded $\sigma(X)$ there is certainly only one solution of (3.3) for sufficiently small $\beta$ since in that case (3.3) considered as a linear equation, $\sigma=1+K(\beta) \sigma,\|K(\beta)\|<1$, for $\beta<\bar{\beta}$ for some positive $\bar{\beta}<\beta_{c}$ [7]. (Similar results hold for the $\varrho$ Eqs. [14].) Hence if there are multiple bounded solutions for $\beta<\beta_{c}$ they must start appearing at some fixed value of $\beta$, say $\tilde{\beta}<\beta_{c}$ and 1 would then be in the spectrum of $K(\beta)$ for $\beta>\tilde{\beta}$. An example in which bounded solutions not defining a state exist was recently found by Brascamp [13].

\section{References}

1. Dobrushin, R.L.: Theory Probability Appl. 13, 197 (1968)

2. Lanford,O.E., Ruelle, D.: Commun. math. Phys. 13, 194 (1969)

3. Lanford,O.E.: Statistical Mechanics and Mathematical Problems, Battelle Seattle 1971 Rencontres. Berlin-Heidelberg-New York: Springer 1973

4. Brascamp, H. J.: Commun. math. Phys. 18, 82 (1970)

5. Ruelle, D.: Statistical Mechanics, New York-Amsterdam: Benjamin 1969

6. Pastur,L. A.: Preprint

7. Gruber, C., Merlini, D.: Physica 67, 308 (1973) 
8. Holley, R.: Commun. math. Phys. 23, 87 (1971)

9. Greenberg, W.: Commun. math. Phys. 22, 259 (1971)

10. Gallavotti, G., Lebowitz, J.: Physica 70, 219 (1973)

11. Ruelle, D.: Commun. math. Phys. 18, 127 (1970).

12. Lebowitz, J.L., Martin-Löf, A.: Commun. math. Phys. 25, 276 (1972)

13. Brascamp, H. J. : to appear in Commun. math. Phys.

14. Gallavotti, G., Miracle-Sole, S.: Commun. math. Phys. 7, 274 (1968)

Communicated by G. Gallavotti

C. Gruber

Laboratoire de Physique Théorique Ecole Polytechnique Fédérale

CH-Lausanne, Switzerland

J. L. Lebowitz

Belfer Graduate School of Science Yeshiva University

New York, N. Y. 10033, USA 